\title{
The Riemannian Structure on the States of Quantum-like Systems
}

\author{
Vittorio Cantoni \\ Politecnico di Torino, I-10129 Torino, Italy
}

\begin{abstract}
A riemannian metric is introduced in the manifold representing the states of a generic physical system, under suitable assumptions of regularity on the "generalized transition probability" defined in [1]. From the mean values of the observables it is then possible to construct gradients and brackets, and in the special case of a system admitting a quantum-mechanical description the latter are shown to be related to the familiar commutators via a skew-symmetric tensor field which is part of the intrinsic geometry of the projective Hilbert space of physical states.
\end{abstract}

\section{Riemannian Structure of the Space of Physical States}

As shown in [1], whenever a physical system is described by a set $S$ of states, a set $O$ of observables, and a function $p(A, \alpha, E)$ representing the probability that the measurement of the observable $A$ on the state $\alpha$ give a result in the Borel set $E$ of the real line (Mackey, [2] axioms I and II), one can construct a function $T(\alpha, \beta),(\alpha, \beta \in S)$, which generalizes the quantum-mechanical transition probability between pure states, and use it to introduce a distance in $S$.

If the preparation of a physical state involves the choice of a finite number of continuous control-parameters, $S$ can reasonably be regarded as an $n$-dimensional manifold in which the control-parameters constitute (or are directly related to) a set of admissible coordinates. This point of view can be extended to systems in which a preparation involves the choice of arbitrary functions by regarding $S$ as an infinitedimensional manifold, which we shall assume to be of class $C^{3}$ and modelled on a real hilbertable vector space ([3], p. 151).

Consider the generic point $\alpha$ of $S$, in the domain $U$ of some chart $(U, \varphi)$, and a neighbouring point $\beta \in U$. Setting $\varphi(\alpha)=P, \varphi(\beta)=Q$, the generalized transition probability $T(\alpha, \beta)$ can be represented by the function $T_{\varphi}(P, Q) \equiv T\left(\varphi^{-1}(P), \varphi^{-1}(Q)\right)$ defined in $E \times E$. Keeping $P$ fixed, $T_{\varphi}(P, Q)$ becomes a function of $Q$ which will be denoted by $T^{P}(Q)$. Recalling the properties of $T(\alpha, \beta)$ established in [1], we have $T^{P}(P)=1$ and $T^{P}(Q)<1$ whenever $Q \neq P$, so that $T^{P}(Q)$ has a maximum at $P$. If $T^{P}(Q)$ 
is assumed to be twice differentiable ([3], p. 6), then the first derivative $D T^{P}(Q)$ vanishes at $P$, i.e. $D T^{P}(P)=0$; consequently the second derivative $D^{2} T^{P}(Q)$, evaluated at $P$, can be identified with a bounded bilinear form on $E \times E$ which determines a symmetric tensor at $\alpha$. If the bilinear form $D^{2} T^{P}(P)$ is non-singular, the associated quadratic form is negative-definite and its opposite determines a metric tensor at $\alpha$, which will be denoted by $\boldsymbol{g}(\alpha)$. Thus under appropriate assumptions of regularity, the function $T(\alpha, \beta)$ determines a riemannian structure on $S([3]$, p. 152). The tensor field $\boldsymbol{g}(\alpha)$ will be assumed to be of class $C^{1}$.

To get further insight on the preceding construction, let us turn our attention to the finite-dimensional case and consider the distance function $d(\alpha, \beta)$ $=\left[2\left(1-T^{1 / 2}(\alpha, \beta)\right]^{1 / 2}\right.$ defined in [1]. Let $x=\left(x^{1}, x^{2}, \ldots, x^{n}\right)$ be the local coordinates defined by the chart $(U, \varphi)$, and set $\varphi(\alpha)=x_{P}, \varphi(\beta)=x_{Q} \equiv x_{P}+d x$. One has $d^{2}(\alpha, \beta)$ $=2\left(1-T^{1 / 2}(\alpha, \beta)\right)=2\left(1-\sqrt{T^{P}(x)}\right)$, and since $T^{P}\left(x_{P}\right)=1$ and $\left.\partial T^{P} / \partial x^{h}\right)_{x P}=0$, $(h=1,2, \ldots, n)$, the first and second terms in the Taylor expansion of $d^{2}(\alpha, \beta)$ at $x_{P}$ vanish, so that the squared distance between the neighbouring points $\alpha$ and $\beta$ is expressed, up to terms of higher order, by the quadratic form

$$
\frac{1}{2} \sum_{h, k} g_{h k} d x^{h} d x^{k}=-\frac{1}{2} \sum_{h, k}\left(\frac{\partial^{2} T^{P}}{\partial x^{h} \partial x^{k}}\right)_{x P} d x^{h} d x^{k} .
$$

The tensorial character of $\boldsymbol{g}$ is then easily checked by preforming a change of local coordinates and taking the vanishing of the first derivatives of $T^{P}$ at $x_{P}$ into account.

\section{Observables, Vector Fields and Brackets}

Let us denote by $\hat{A}(\alpha)$ and $\hat{B}(\alpha)$ the mean values of two observables $A$ and $B$ on the state $\alpha$. Like all the entities previously introduced, $\hat{A}(\alpha)$ and $\hat{B}(\alpha)$ can be derived from the probability function $p(A, \alpha, E)$, which is itself operationally determinable.

If $\hat{A}(\alpha)$ and $\hat{B}(\alpha)$ are of class $C^{2}$ as functions of $\alpha$ on the riemannian manifold $S$, their gradients $\operatorname{grad} \hat{A}$ and $\operatorname{grad} \hat{B}([3]$, p. 163) are well-defined, together with the bracket $[\operatorname{grad} \hat{A}, \operatorname{grad} \hat{B}]([3]$, p. 104). With our present assumptions there is no warrantee that $[\operatorname{grad} \hat{A}, \operatorname{grad} \hat{B}]$ will be related to the gradient of a function $\hat{C}(\alpha)$ representing the mean value of some observable $C$; but if this is the case, the structure determined by the bracket operation can be transported back from the vector fields to the observables.

Though derived from the mean values of $A$ and $B$, the bracket is implicitly related to the actual probability distributions of the observables via the riemannian metric.

In the next section we shall show that in the case of a quantum-mechanical system the structure just considered is related to the familiar one determined by the commutators of the hermitian operators representing the observables in Hilbert space.

\section{Connection with Quantum Mechanics}

Let us denote by $H$ the complex Hilbert space of a quantum-mechanical system. The manifold $S$ of our general scheme now coincides with the projective space $\tilde{H}$ associated with $H$, regarded as a real manifold. Let $\omega \in \tilde{H}$ represent a generic 
physical state. We shall introduce in $H$ an orthonormal basis $\left\{\boldsymbol{\varepsilon}_{0}, \boldsymbol{\varepsilon}_{H}\right\}$ $\equiv\left\{\varepsilon_{0}, \varepsilon_{1}, \varepsilon_{3}, \ldots\right\}$ whose elements are labeled, for future convenience, with the index ${ }_{0}$ and with odd positive integers, and such that $\varepsilon_{0}$ coincides with one of the unit vectors of $H$ representing the state $\omega$. Thus the index ${ }_{H}$ (and all capital indices) runs over a finite set of positive odd integers if $H$ is finitedimensional, and over the set of all positive odd integers if $H$ is infinitedimensional. Let $U_{\omega}$ be the neighbourhood of $\omega$ constituted by the states $\alpha$ such that $T(\omega, \alpha)>0$, so that each element of $U_{\omega}$ is represented in $H$ by vectors with a nonvanishing component of index ${ }_{0}$. Disposing of the arbitrary complex factor in the Hilbert representation of the physical states, we associate with each element $\alpha \in U_{\omega}$ the uniquely determined unit representative vector $\alpha \equiv \alpha^{0} \boldsymbol{\varepsilon}_{0}+\sum_{H} \alpha^{H} \boldsymbol{\varepsilon}_{H}$ whose component $\alpha^{0}$ is positive. Setting $\alpha^{0} \equiv x^{0}$, $\alpha^{H}=x^{H}+i x^{H+1}\left(x^{H}, x^{H+1}\right.$ real $)$ one has $|\alpha|^{2}=\left(\alpha^{0}\right)^{2}+\sum_{H} \bar{\alpha}^{H} \alpha^{H}=\left(x^{0}\right)^{2}+\sum_{h}\left(x^{h}\right)^{2}=1$, (where $h$, like all the lower-case indices, runs over the set of all positive indices from 1 to $n$ or from 1 to $\infty$, according to the dimension of $H$ ). The correspondence $\alpha \rightarrow\left\{x^{h}\right\} \equiv\left\{x^{1}, x^{2}, \ldots\right\}$ defines a local coordinate system in $U_{\omega}$, while $x^{0}=\left[1-\sum_{h}\left(x^{h}\right)^{2}\right]^{1 / 2}$ is a dependent quantity.

The generalized transition probability, which now coincides, as shown in [1], with the usual squared modulus of the scalar product, is given by $T(\alpha, \beta)=\mid \alpha^{0} \beta^{0}$ $+\left.\sum_{H} \bar{\alpha}^{H} \beta^{H}\right|^{2}$, and can be regarded as a function of the independent coordinates $\left\{\alpha^{h}\right\}$ and $\left\{\beta^{h}\right\}$ of the states $\alpha$ and $\beta$ in $U_{\omega}$. In the finite-dimensional case the components $g_{h k}$ of the metric tensor at $\alpha$ can readily be computed by means of Equation (1), and have the form

$$
g_{h k}=\delta_{h k}+\frac{x^{h} x^{k}}{1-\sum_{l}\left(x^{l}\right)^{2}},
$$

and it is easy to verify that the quadratic form $\sum_{h k} g_{h k} d x^{h} d x^{k}$ makes sense even in the infinite-dimensional case and represents the squared modulus of the vector

$$
-\frac{x^{0}}{\sqrt{\sum_{h} x^{h} d x^{h}}} \boldsymbol{\varepsilon}_{0}+\sum_{H}\left(d x^{H}+i d x^{H+1}\right) \boldsymbol{\varepsilon}_{H}
$$

which is orthogonal to $\alpha$ in $H$ and can therefore be regarded as tangent to $S$ at $\alpha$, if $U_{\omega}$ is identified with the appropriate portion of the surface of the unit Hilbert sphere.

The matrix $\boldsymbol{g}_{h k}$ has the inverse $\boldsymbol{g}^{h k}=\delta^{h k}-x^{h} x^{k}$, from which the gradients and the brackets can be explicitly constructed. If $\varphi$ is a covariant vector field in $\tilde{H}$ with components $\varphi_{h}$, we shall denote by $\boldsymbol{g}^{\dagger} \boldsymbol{\varphi}$ the contravariant vector field $\boldsymbol{v}$ with components $g^{h k} \varphi_{k}$. Conversely we shall write $\varphi=\boldsymbol{g}_{\tilde{\imath}} \boldsymbol{v}$.

Besides the riemannian metric, the manifold $\tilde{H}$ possesses a skew-symmetric tensor field which is also determined by the complex scalar product of $H$. In fact, if 
$H$ is regarded as a real vector space with basis $\left\{\boldsymbol{e}_{0}, \boldsymbol{e}_{h}\right\} \equiv\left\{\boldsymbol{\varepsilon}_{0}, \boldsymbol{\varepsilon}_{1}, i \boldsymbol{\varepsilon}_{1}, \boldsymbol{\varepsilon}_{2}, i \boldsymbol{\varepsilon}_{2}, \ldots\right\}$, then the imaginary part of the original scalar product is a bilinear form associated with the skew-symmetric tensor $\sum_{H}\left(\boldsymbol{e}_{H} \otimes \boldsymbol{e}_{H+1}-\boldsymbol{e}_{H+1} \otimes \boldsymbol{e}_{H}\right)$, which induces in the manifold $\tilde{H}$ at $\omega$ the skew tensor $\boldsymbol{\eta}(\omega)$ with components $\eta_{h k}$ equal to 1 if $h$ is odd and $k=h+1$, to -1 if $h$ is even and $k=h-1$, to zero otherwise. It is easily checked by passing to a new basis $\left\{\boldsymbol{\varepsilon}_{0^{\prime}}, \boldsymbol{\varepsilon}_{\boldsymbol{H}^{\prime}}\right\}$ in $H$ (and keeping $\omega$ fixed, so that $\left.\boldsymbol{\varepsilon}_{0^{\prime}}=\exp (i \theta) \boldsymbol{\varepsilon}_{0}\right)$, and by performing the associated coordinate transformation in $\tilde{H}$, that our construction of the tensor $\boldsymbol{\eta}(\omega)$ is basis-independent, so that the skew-field $\boldsymbol{\eta}$ obtained by repeating the construction at each point is an intrinsic geometric element of $\tilde{H}$. If $\varphi$ is a covariant vector field in $\tilde{H}$, with components $\varphi_{h}$, we shall denote by $\eta^{\uparrow} \varphi$ the contravariant vector field with components $\eta^{h k} \varphi_{k}$, where $\left(\eta^{h k}\right)$ is the matrix inverse to $\left(\eta_{h k}\right)$.

Consider now the observables $A$ and $B$, represented in $H$, according to the quantum-mechanical description, by two hermitian operators $\boldsymbol{A}$ and $\boldsymbol{B}$. Set $\boldsymbol{C}=-2 i(\boldsymbol{A B}-\boldsymbol{B} \boldsymbol{A})$. The mean value $\hat{A}(\alpha)$ is given by

$$
\begin{aligned}
\hat{A}(\alpha)= & A_{00}\left(x_{0}\right)^{2}+x^{0} \sum_{H}\left[A_{H 0}\left(x^{H}-i x^{H+1}\right)+A_{0 H}\left(x^{H}+i x^{H+1}\right)\right] \\
& +\sum_{H k} A_{H k}\left(x^{H}-i x^{H+1}\right)\left(x^{k}+i x^{k+1}\right)
\end{aligned}
$$

where $\left\{A_{00}, A_{0 H}, A_{H 0}, A_{H k}\right\}$ are the matrix elements of $\boldsymbol{A}$ in the basis $\left\{\boldsymbol{\varepsilon}_{0}, \boldsymbol{\varepsilon}_{\boldsymbol{H}}\right\} . \hat{B}(\alpha)$ and $\hat{C}(\alpha)$ have analogous expressions.

It is now possible to verify the equation

$$
[\operatorname{grad} \hat{A}, \operatorname{grad} \hat{B}]=-\boldsymbol{\eta}^{\uparrow} \boldsymbol{g}^{\downarrow} \operatorname{grad} \hat{C} .
$$

This is most easily done, in our coordinate system, by computing the $h$-th components at the point $\omega$ (which had been chosen arbitrarily in $S$ ). It must be remembered that $x^{0}$ is not an independent coordinate in $U_{\omega}$.

Equation (3) establishes the relation between the bracket operation considered in the more general context of the previous sections and the quantum-mechanical commutators.

\section{Remarks}

If $S$ is the phase space of a classical system our construction fails, because $T(\alpha, \beta)$, regarded as a function of its second argument, is then equal to zero everywhere except at the point $\alpha$ where, therefore, it is not differentiable. Notice however the analogy between the symplectic structure of classical phase space and the skewsymmetric tensor field of $\tilde{H}$. The latter can be used alone (i.e. independently of $\boldsymbol{g}$ ) to define a Poisson-bracket operation $[\hat{A}, \hat{B}]$ from the mean values of the observables, exactly in the same way as the former is used to form Poisson-brackets from the mean (but then also exact) values of the observables in classical phase-space. It is possible to verify $([4])$ that $[\hat{A}, \hat{B}]=\hat{C}$ in $\tilde{H}$, where, as above, $C$ is the observable described by the hermitian operator $-2 i(\boldsymbol{A B}-\boldsymbol{B} \boldsymbol{A})$ in $H$. 
The explicitation of the skew tensor field $\boldsymbol{\eta}$ in $\tilde{H}$ should be of some help towards a better understanding of the connection between classical and quantum mechanics: in particular it suggests that it should in some sense be possible to regard the classical phase space as the limit of a projective Hilbert space in which the metric $\boldsymbol{g}$ (but not the skew-field $\boldsymbol{\eta}$ ) becomes degenerate, the degeneracy being related, on account of the definition of $T(\alpha, \beta)$, to the existence, for every pair of states $\alpha$ and $\beta$, of at least one observable with non-overlapping ranges of values on $\alpha$ and $\beta$.

\section{References}

1. Cantoni, V.: Commun. math. Phys. 44, 125 (1975)

2. Mackey,G.W.: Mathematical foundations of quantum mechanics. New York: Benjamin 1963

3. Lang, S.: Differential manifolds. Reading: Addison Wesley 1972

4. Cantoni, V.: Intrinsic geometry of the quantum-mechanical "phase space", Hamiltonian systems and correspondence principle. Rend. Accad. Nazl. Lincei, to appear

Communicated by R. Haag

Received June 6, 1977 
